\title{
Study of idiopathic inflammatory myopathies with special reference to borderland between idiopathic inflammatory myopathies and muscular dystrophies
}

\author{
Satish V. Khadilkar, Shekhar G. Patil' ${ }^{1}$, Sanjeev N. Amin ${ }^{2}$ \\ Department of Neurology, Grant Medical College and Sir JJ Group of Hospitals, Mumbai, ${ }^{1}$ Kokilaben Dhirubhai Ambani Hospital, Andheri, \\ Mumbai, ${ }^{2}$ Bombay Hospital Institute of Medical Sciences, Mumbai, India.
}

\begin{abstract}
Background: Idiopathic inflammatory myopathies (IIMs) form important treatable myopathies, hence it is important to recognize and categorize them. In some cases, the differential diagnosis between IIM and muscular dystrophies can be difficult. Aim: To study the clinical and laboratory features of patients with IIMs and compare and contrast this group with limb girdle muscular dystrophies (LGMDs). Setting and Design: A prospective study for the period of five years [1999-2004] was undertaken at a tertiary neuromuscular center. Materials and Methods: Bohan and Peter criteria were used for the diagnosis of IIM and Bushby criteria were used for the diagnosis of LGMD. Patients underwent history, clinical examination, hematological tests, electrophysiological studies and muscle biopsy. The biopsies were studied for histology and immunocytochemistry. A clinical scoring system was evolved to differentiate IIM from LGMD and was validated in a blinded manner. Receiver operator curves were used as the statistical method to analyze the sensitivity and specificity. Results and Conclusions: In the IIM group, dermatomyositis was most common, followed by polymyositis, occurring in young females. Overlap group was less common. In patients with polymyositis, onset in upper girdle was associated with adverse outcome. The scoring system helped to differentiate IIM from LGMD, mainly using clinical pointers. This was particularly valuable in chronic cases.
\end{abstract}

Key words: Inflammatory myopathies, muscular dystrophy, scoring system

\section{Introduction}

Idiopathic inflammatory myopathies (IIMs) are characterized by prominent muscle weakness often accompanied by systemic manifestations, which on histology show inflammation of the skeletal muscle.
This group consists of three major subsets, namely dermatomyositis (DM), polymyositis (PM) and inclusion body myositis (IBM) ${ }^{[1-2]}$ The clinical relevance of this group lies in its response to therapy. Hence it is important to make an accurate diagnosis of these conditions. In the last few years, the body of evidence seems to suggest that the frequency of occurrence of PM is much less than has been believed and the borderlands between the inflammatory and other types of myopathies are being explored and clarified with the advent of newer investigative techniques. Literature review suggests that a proportion of reported cases of PM are in fact, IBM, myositis associated with connective tissue disorders, dystrophies with inflammation or DM. ${ }^{[1]}$ Historically PM has been differentiated from dystrophies on the basis of clinical and histological findings, however, despite careful evaluation, such differentiation into one of these groups may not be possible in all cases. In India, limb girdle muscular dystrophies (LGMDs) are common and sporadic cases lead to diagnostic dilemma. ${ }^{[3]}$ The limited access to modern hematological, immunological and histological tests adds to the diagnostic difficulty.

Hence we have undertaken this study to evaluate the clinical and laboratory features of inflammatory myopathies, compared and contrasted them with muscular dystrophies and evolved a clinical scoring system to help the clinician arrive at a diagnosis.

\section{Materials and Methods}

This study was carried out in a neuromuscular clinic of a tertiary center. Information collected from two neurology and one rheumatology centers was analyzed. The study period was 1999 to 2004. Informed consents in the languages understood by patients were obtained and the study design was approved by the hospital 
board. Information was collected on the following points.

\section{Clinical features}

- Age at onset

- Duration of the disease

- Girdle of Onset (either shoulder or hip)

- Evolution of disease

- The time taken for weakness to progress from the girdle of onset to the unaffected girdle

- Differential weakness in at least three groups of muscles.

- Difference of at least one MRC power grade among the group muscles acting at the same joint (e.g. Quadriceps and hamstrings, adducters and abducters of hip)

- Distal weakness

- Dysphagia

- Weakness in the neck flexor group of muscles.

\section{Associated features}

Pain, Fever, Swelling, Rash, Weight loss, Fatigue, Malignancy

\section{Laboratory Investigations}

CK, ESR, ANA, dsDNA, other serological markers as indicated

\section{Histopathology}

Fiber size variability, Hypertrophic fibers, Basophilic/ Rounded fibers, Myophagia, Interstitial Inflammation, Necrotic fibers, Muscle fiber splitting, Internalized nuclei.

Immunocytochemistry for dystrophin I, II, III, alpha, beta gamma and delta sarcoglycans and dysferlin

\section{Inclusion and exclusion criteria}

- Inflammatory myopathy

Inclusion: Patients satisfying the Bohan and Peter criteria $^{[4]}$ for inflammatory myopathy: Presence of three out of five criteria makes the diagnosis of probable inflammatory myopathy.

The patients were subclassified as DM, PM, Juvenile DM, Overlap syndromes and malignancy-associated IIM and IBM.

Exclusion:

Any patient with family history of myopathic illness

Patients with history of use of known myotoxic drugs

- Limb girdle muscular dystrophy

Inclusion: Patients fulfilling the Bushby clinical criteria $^{[5]}$ and having either immunocytochemical or genetic confirmation of the dystrophy and/or having more than one family member affected with dystrophy.
Exclusion: Congenital onset, ptosis or weakness of ocular muscles, onset in facial muscles and a rash compatible with dermatomyositis. ${ }^{[5]}$

Using the clinical and laboratory features, we devised a scoring system based on the STUDY GROUP, which consisted of randomly selected 25 patients each from the group of IIM and LGMD. The score was then applied to a VALIDATION GROUP of 25 patients each, in a blinded manner to validate the sensitivity of the score. After validation, the score was applied to a select group of 18 patients [nine from each group] in whom the disease duration was between one to three years [as this duration poses maximal difficulty in diagnosis]. The utility of using this score in classifying patients to a particular group was studied. The scoring system is shown in Table 1.

\section{Results}

\section{Preliminary data}

A total of 61 consecutive patients with inflammatory myopathy were studied. Patients with DM formed the largest subgroup accounting for 34 cases. Of the remaining, 18 had PM, and nine patients were cases of inflammatory myopathy associated with connective tissue disease. The male: female ratio in the entire group was 1:3 (20:61). In the PM group only three patients were males. The mean age of the inflammatory myopathy group was 34.3 years (7-56 years) with a mean duration of illness being 1.08 years.

The group of muscular dystrophy was a select group of 61 patients in whom there was a confirmatory diagnosis of a muscular dystrophy either by familial nature or by abnormalities of immunostaining for dysferlin and sarcoglycans. The mean age of this group was 23.71 years (8-52 years) with a mean duration of illness at presentation being 6.3 years.

\section{Clinical profile}

The group of inflammatory myopathy had rapid

\begin{tabular}{|c|c|c|c|}
\hline \multicolumn{4}{|c|}{ Table 1: Scoring system } \\
\hline Feature & & Score & \\
\hline Age at onset (years) & $<20: 1$ & $>20: 2$ & \\
\hline Duration of disease & $<6$ mths: 3 & 6-24 mths:2 & $>2$ yrs:1 \\
\hline Evolution of disease & & & \\
\hline [one girdle to the other] & $<6$ mths: 3 & 6-24 mths:2 & $>2$ yrs: 1 \\
\hline Onset & Shoulder girdle:1 & Pelvic girdle:0 & \\
\hline Differential weakness & Present:0 & Absent:2 & \\
\hline Distal weakness & Present: 1 & Absent:2 & \\
\hline Dysphagia & Present:2 & Absent:0 & \\
\hline Hypertrophy & Present:0 & Absent:1 & \\
\hline Atrophy & Present:0 & Absent:1 & \\
\hline & Absent:0 & $\leq 2$ symptoms: 1 & \\
\hline Associated Features & $3 \leq 7$ & & \\
\hline Lab Investigations & ESR > 20:1 & and < 20: 0 & \\
\hline
\end{tabular}


evolution of the disease. The mean duration of spread of the disease from one girdle to other was 0.45 years. In four patients, the disease remained restricted to the girdle of onset till the time of their evaluation. In 55 patients, the disease began in the pelvic girdle. In the remaining six patients, the weakness started in the shoulder girdle. All six patients, whose disease began in the upper limbs, had PM.

In addition to the proximal weakness in the group of inflammatory myopathy, 14/61 (22.95\%) patients had distal weakness. Nearly $50 \%$ of patients [8/18] with PM had demonstrable distal weakness. All six patients, whose disease started in the shoulder girdle and in addition patients with rapid evolution of the disease had distal weakness. Dysphagia was seen in 15 patients and was seen early in the disease course in five patients. Differential weakness was exceptional and seen only in two patients.

Figure 1 depicts the frequency of occurrence of constitutional symptoms, the commonest being myalgias.

\section{Dermatomyositis \\ Adult DM}

Adult DM was the largest subgroup with 28/61 $(45.9 \%)$ patients. The sex ratio showed slight female preponderance of 1:1.15. The mean age at presentation was 37.02 years. The mean duration of illness of 1.06 years, was slightly more than the PM group. The disease had onset in the hip girdle in all the patients. There was spread of the disease to involve the shoulder girdle in 26 patients after a mean duration of 0.43 years. In the remaining two patients the disease was restricted to the hip girdle. The mean CK value was 2476.3 IU.

\section{Juvenile DM}

Six cases (four males and two females) of juvenile DM were seen. Mean age at presentation was 8.2 years. They became symptomatic at approximately five to six years or 11-12 years. Skin rash was seen or its history obtained in three patients at the time of their first evaluation by us. All patients had proximal weakness involving the hip and shoulder girdle. Calcinosis was seen in two

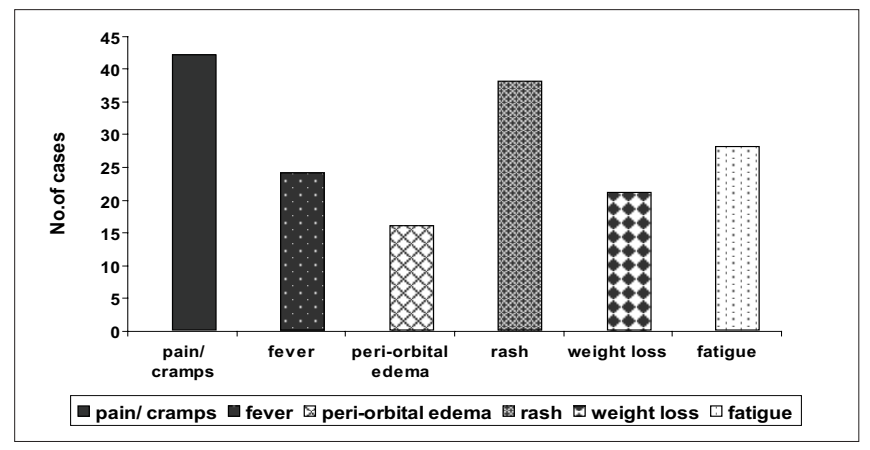

Figure 1: Constitutional symptoms patients, in whom the time lag in seeking medical advice was more than two years. Skin ulceration was also seen in these two patients with calcinosis. Differential weakness was not seen in any patient of this group. Six patients showed evidence of distal muscle weakness. All these patients were cases of juvenile DM. Dysphagia was seen in approximately one-fourth patients in the group. Mean CK value was 2765 IU.

The commonest constitutional symptom was skin rash followed by muscle aches, pains and cramps. Nearly half the patients had significant weight loss and were disabled because of sensation of fatigue.

\section{Polymyositis}

This group comprised 18 patients. The male to female ratio was 1:6 in this group. The mean age at presentation for this group was 36.7 years. The disease course was of shorter duration in comparison with patients of DM, with a mean of 0.89 years; $55.5 \%$ of the patients [10/18] had disease duration of more than six months. The disease spread from one girdle to the other in 16 patients after a mean duration of 0.42 years. Of the constitutional symptoms rash was not seen in any of the patients in the PM group. Differential weakness was seen in two patients only. Eight patients had demonstrable distal weakness. Distal weakness was seen in patients with rapid evolution of disease or in those in whom the onset of disease was in the shoulder girdle. One single patient with cervical malignancy-associated PM was a part of this group. As with patients of DM, nearly half the patients had significant weight loss and were disabled because of sensation of fatigue and generalized weakness.

Six patients had disease onset in the shoulder girdle. All these patients had severe involvement of the lungs in the form of interstitial fibrosis, documented by chest X-ray and computed tomography of the chest. The severe involvement of the lungs necessitated ventilatory care in two patients. Poor clinical course was the hallmark of all these patients. One patient expired in the hospital. The mean CK value was 2134 IU.

\section{Muscular dystrophy}

This group included 14 patients with dysferlinopathy [mean age 19.9years] ${ }^{[6]} 25$ were cases of sarcoglycanopathy [mean age 25.4 years], ${ }^{[7]}$ [both published series] and the remaining 22 were unclassified muscular dystrophies. Nine patients with dysferlinopathy had the onset of disease in the distal muscles of the lower limbs. In all the remainder the girdle of onset was the hip musculature. At the time of presentation to us, 45/61 $(73.7 \%)$ patients had demonstrable weakness in the distal musculature. Thirty patients had hypertrophied muscles while another 38 showed atrophy in some muscle groups. In all, 12 patients with dystrophy showed hypertrophy of some muscle groups and at 
the same time had atrophied muscles at other areas. Of these 61 patients only three patients did not have either hypertrophied or atrophied muscles. Except for pain/ cramps in five patients none of the other constitutional symptoms were seen in this group. The mean CK value for the dysferlinopathy group was $5847 \mathrm{IU}$, for sarcoglycanopathies 2346.4 IU, and was 2488.6 IU for the group of dystrophies as a whole.

Comparison between IIM and MD: The disease showed a slow progression in the MD group and the involvement of the initially unaffected girdle occurred after a mean duration of 3.52 years. In almost all patients with inflammatory myopathy the disease had spread from one girdle to other within a span of six months. The total duration of illness showed marked variation in both the groups. Dysphagia was seen only in the group

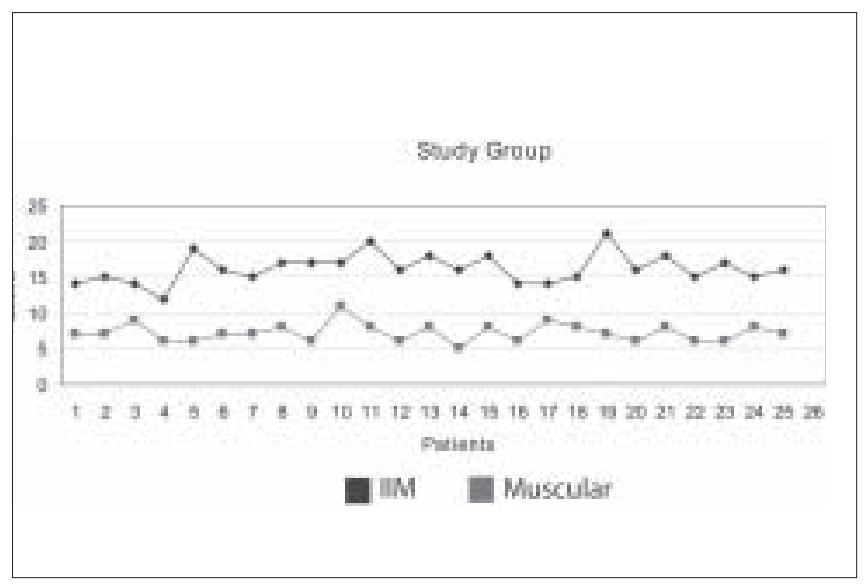

Figure 2: Scoring system: Study Group [Muscular Dystrophy, IIM] Receiver Operator Curves were used, giving

priority to specificity. At 14 , the sensitivity for IIM was $96 \%$ and specificity for IIM was $100 \%$. At 8 , the sensitivity for dystrophy was $84 \%$ and the specificity was $100 \%$

\begin{tabular}{lcc}
\hline Table 2: Clinical profile of patients matched for duration 1-3 years \\
\hline & $\begin{array}{c}\text { Inflammatory } \\
\text { myopathy }\end{array}$ & $\begin{array}{c}\text { Muscular } \\
\text { dystrophy }\end{array}$ \\
Mean age (years) & 35.44 & 19.89 \\
Mean disease duration (years) & 1.55 & 1.5 \\
Mean duration for & 0.55 & 0.91 \\
evolution of disease (years) & 1 & 7 \\
Patients with differential weakness & 4 & 7 \\
Patients with distal weakness & 2 & 0 \\
Patients with dysphagia & 9 & 0 \\
Patients with Constitutional symptoms & 14.3 & 7.7 \\
Mean Score & &
\end{tabular}

of inflammatory myopathy. Distal weakness was seen in 16/61 (26.2\%) patients with inflammatory myopathy. Nearly $75 \%$ of patients with dystrophy had distal weakness. Hypertrophy was not seen in patients with inflammatory myopathy, while atrophy was noticed in three patients only.

\section{Scoring system}

The mean score in the study group of 25 patients with inflammatory myopathy was $16.2 \pm 2.08$. The mean score in the study group of 25 patients with muscular dystrophy was $7.06 \pm 1.32$.

Based on these values, a patient scoring less than 8 was labeled as dystrophy and a score of 14 and above was interpreted as inflammatory myopathy. The patients with score of 9-13 constituted the grey zone. On blinded application of the score on validation group of 25 patients each, the specificity and sensitivity was found to be same as that of the study group [Figures 2 and 3].

We compared patients within both these groups, who were matched for disease duration of one to three years. The findings in this matched group are shown in Table 2.

\section{Investigations}

The serum levels of CK and calcium were tabulated; CK levels were raised in all the patients [640 IU to 8846 IU].The CK elevation did not form a differentiating point between IIM and muscular dystrophies. Patients with DM had a low serum calcium level at the peak of their disease, which normalized once they received steroids and other immunosuppressive agents.

\section{Muscle biopsy}

All patients were subjected to muscle biopsy. The patients in the MD group were further subclassified into groups of dysferlinopathy, sarcoglycanopathy and

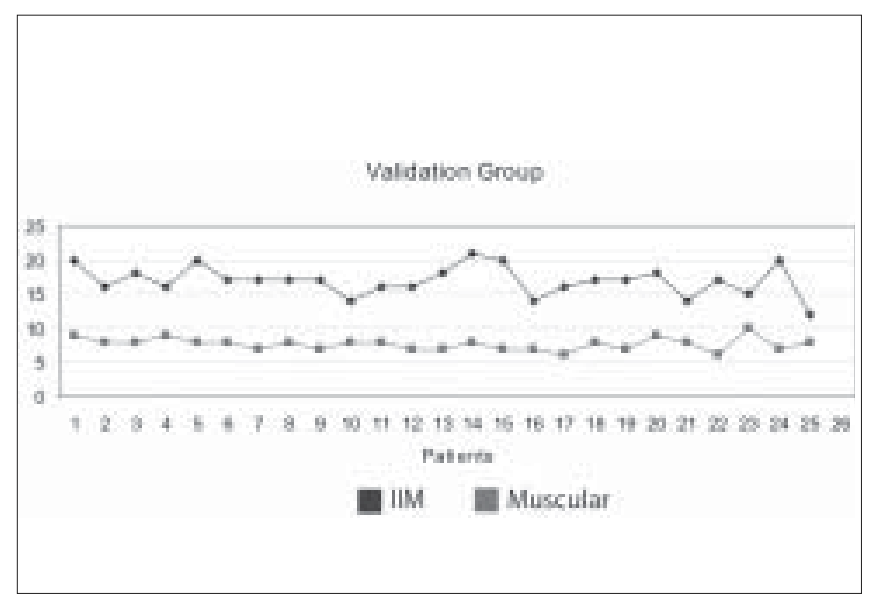

Figure 3: Scoring system: Validation Group [Muscular Dystrophy, IIM]

others based on immunocytochemistry reports. Table 3 shows histopathological findings in inflammatory myopathies.

\section{Discussion}

\section{Preliminary data}

The mean age of patients with inflammatory myopathies in the present study was 34.3 years which is similar to other studies from India and other parts of 


\begin{tabular}{lccc}
\hline & Table 3: Histopatholgical findings & \\
\hline & Polymyositis & $\begin{array}{c}\text { Dermatomyositis } \\
\mathbf{N}=\mathbf{3 4}\end{array}$ & $\begin{array}{c}\text { Associated with Connective tissue } \\
\text { disease } \mathbf{N = 9}\end{array}$ \\
Abnormal biopsy & $\mathbf{N = 1 8}$ & 29 & 6 \\
Necrotic fibers & 15 & $n o n e$ & $4 / 6$ \\
Regenerating fibers & $14 / 15$ & $1 / 29$ & $3 / 6$ \\
Interstitial inflammation & $10 / 15$ & $5 / 6$ & None \\
Perifascicular atrophy & $12 / 15$ & $24 / 29$ & $3 / 6$ \\
Vasculopathy & None & $22 / 29$ & \\
\hline
\end{tabular}

the world. ${ }^{[8-14]}$ The frequency of subgroups was DM, PM, overlap syndromes, JDM and malignancy-related IIM in decreasing order of frequency. Studies using Bohan and Peter's criteria have yielded more or less similar pattern of subgroups of IIM..$^{[8-14]}$ Bohan and Peter's criteria are widely accepted and the sensitivity of these criteria ranges from $74-100 \%$ in large series.${ }^{[15]}$ However, studies taking the help of modern serological tests demonstrate higher frequency of the overlap syndromes, in patients who would be classified as PM or DM using the clinical criteria. ${ }^{[16-17]}$ In the 10 patients in the present series, we had information on the serology and one patient changed class from DM to overlap syndrome.

Adult dermatomyositis [DM] was the most common IIM. This is in keeping with studies in the world literature. ${ }^{[6,8,9] .}$ There is comparatively less information from India and few studies are available for comparison. In the study by Porkodi et al., primary DM was seen in $29.8 \%$. In the present study, this group was relatively homogenous, patients presenting with skin rash, or periorbital edema, myalgia and fatigue. Even though adult DM formed the largest group, a longitudinal clinical and serological follow-up of these patients will be necessary to study evolution to more widespread connective tissue disease.

\section{Juvenile dermatomyositis}

This group had mild male predominance. The male dominance in JDM seems to be consistently seen in other Indian, Japanese and Saudi Arabian studies..$^{[8-11,14]}$ The western literature on JDM has documented female preponderance. ${ }^{[18]}$ This observation is curious and difficult to explain. The bimodal age at onset without sex preference, noted earlier, was seen in our patients. ${ }^{[18]}$ The prominent clinical features were skin rash and proximal muscle weakness without any involvement of the gastrointestinal (GI), neurological, and respiratory systems. Calcinosis was resistant to therapy in one of the two patients.

Lipodystrophy and associated metabolic abnormalities are reported in $20-25 \%$ of patients with JDM. The metabolic abnormalities like hypertriglyceridemia and insulin resistance ${ }^{[16]}$ were not encountered in the present study and only one patient had lipodystrophy.

\section{Polymyositis}

The high incidence of PM [30\%] in the present study is noteworthy. Our patients were younger, had symmetrical, mainly proximal weakness, CK elevation more than $1000 \mathrm{IU}$ and showed response to therapy. These patients have had muscle biopsies specifically looking for inclusions which were not seen in any of them. Hence we feel that these patients represent true PM and not IBM. These findings are of interest as there has been a growing body of evidence pointing towards the rarity of true PM. ${ }^{[1,19]}$ The literature suggests that follow-up examinations have seen cases of PM at older ages turning to IBM, with demonstration of inclusions and development of asymmetric distal weakness. We, however, feel that true PM exists and formed $30 \%$ of cases of IIM in the present study. These patients will need further long-term evaluation for newer clinical and laboratory features. ${ }^{[20,21]}$ MHC I antibody studies were not feasible due to setup limitations.

We wish to highlight a subgroup of six patients of PM who had the onset of disease in shoulder girdle, rapidly followed by dysphagia and respiratory muscle involvement. Interstitial fibrosis was seen in four of these six patients. These patients did not respond well to steroids, other immunosuppressants or intravenous immunoglobulins, and were disabled due to persistent dysphagia and upper girdle weakness. The combination of respiratory muscle involvement and interstitial fibrosis resulted in ventilator dependency in two and one patient succumbed while on ventilator. Thus, the prognosis was adverse in these patients. The interstitial lung disease, which is a well-recognized adverse prognostic feature, went hand in hand with the rapid upper limb onset and dysphagia pointing to adverse outcome. In some respects, these patients are similar to those described by Pestronk et al., ${ }^{[22]}$ as brachiocervical inflammatory myopathies. Predominant upper limb weakness and dysphagia are the common clinical denominators. However, steroid responsiveness has not been a feature of our patients. We plan further serological information on this group.

Distal weakness was seen in $22 \%$ of patients of IIM and in eight out of 18 patients with PM. Studies from the literature have demonstrated a similar observation. The distal weakness was seen particularly in patients with rapidly evolving disease and in those with shoulder 
girdle onset. In all patients, it was milder than the proximal weakness.

\section{Overlap Group}

Nine patients were classified in this subgroup of IIM. Serology in all the patients helped to confirm their classification. In addition one single patient of DM was reclassified as overlap syndrome after using the lab criteria. This group may be under-represented and repeated follow-ups over time may be necessary to judge its actual prevalence.

\section{Malignancy}

The association of malignancy with $\mathrm{DM}^{[23]}$ has strong support in the literature. The association of malignancy with PM in not as strong but is definitely higher than the general population. In the present study, a single patient of PM was identified, who had malignancy of the cervix. Patients of IIM have the highest risk of suffering from cancer in the first five years after the diagnosis of the disease, as malignancy may precede, accompany or follow the muscle weakness. Hence this figure may also grow with follow-up studies.

\section{Scoring system and the Differential diagnosis}

It is a common clinical experience that patients of inflammatory myopathy, especially PM can be confused with patients of LGMD in general and dysferlinopathy in particular, because of the prominent inflammatory cells seen on the muscle biopsy specimens. Differentiation between acute PM and LGMD does not pose a difficulty, but when patients present for the first time with duration of illness longer than one year, there is a strong likelihood of confusion. Differentiation based on the muscle biopsy findings is at times difficult. ${ }^{[24]}$ This is exemplified by the fact that 11 of our patients with muscular dystrophies had received corticosteroids with the presumptive diagnosis of inflammatory myopathy. Initiation of steroids in patients with LGMD may not only be ineffective but has shown to increase the weakness. Such loss of power cannot be regained after stopping the steroids. ${ }^{[25,26]}$ Dalakas $^{[21]}$ has suggested formulation of new criteria to help distinguish PM from muscular dystrophies. The molecular profiles as suggested by Gallardo ${ }^{[27]}$ can be used to differentiate IIM from muscular dystrophy. These proposed guidelines rely on modern serological and immunocytochemical techniques.

Our scoring system uses clinical criteria; as sophisticated laboratory facilities are limited in the Indian setting. Scoring system can successfully differentiate IIM from LGMD with a sensitivity of over $80 \%$. Thus, it can form an important guide for the clinician, when modern tests are not at hand. This is particularly relevant in cases that are chronic. We have chosen an outer limit for comparison at three years because none of our patients with PM/IIM had a disease course of more than three years and differentiation of patients with disease duration less than one year is relatively easy. The scoring system may have a limited ability in patients of IBM as these patients share the chronicity with muscular dystrophy.

\section{Conclusions}

Dermatomyositis in adults was the most frequent IIM. Polymyositis formed the second common IIM, occurring in young adult females. The scoring system helped differentiate PM from LGMD when the duration was chronic. This clinically oriented scoring system of differential diagnosis is particularly relevant in the Indian situation, where laboratory facilities are limited.

\section{References}

1. Amato AA, Griggs RC. Unicorns, dragons, polymyositis and other mythological beasts. Neurology 2003;61:288-9.

2. Amato AA, Barohn RJ. Idiopathic inflammatory myopathies. Neurol Clin 1997;15:615-48.

3. Khadilkar SV. LGMD in Mumbai region, India. In: Burgress VN, editor. Trends in muscular dystrophy research. 1st ed. USA: Nova Science; 2005. p. 32-59.

4. Bohan A, Peter JB. Polymyositis and dermatomyositis: Part 1. N Engl J Med 1975;292:344-7.

5. Bushby KM. Limb girdle muscular dystrophy. In: Emery AE, editor. Diagnostic criteria for neuromuscular disorders. 3rd ed. The Netherlands: Baarn; 1994. p. 25-31.

6. Khadilkar SV, Singh RK, Kulkarni KS, Chitale AR. A study of clinical and laboratory features of 14 Indian patients with dysferlinopathy. J Clin Neuromusc Dis 2004;6:1-8.

7. Khadilkar SV, Singh RK, Katrak SM. Sarcoglycanopathies: A report of 25 cases. Neurol India 2002;50:27-32.

8. Porkodi R, Shanmuganandan K, Parthiban M, Madhavan R, Rajendran P. Clinical spectrum of inflammatory myositis in South India: A ten year study. J Assoc Physicians India 2002;50:1255-8.

9. Prasad ML, Sarkar C, Roy S, Bagchi U, Singh RR, Singh YN, \& al. Idiopathic inflammatory myopathy: Clinico pathological observations in the Indian population. Br J Rheumatol 1992;31:835-9.

10. Thussu A, Prabhakar S, Bansal SK, Sawhney IMS, Chopra JS. Polymyositis: A clinical profile. Neurol India 1993;41:93-7.

11. Gupta SJ. The idiopathic inflammatory myopathies: Current perspectives and management. J Indian Rheumatol Assoc 2004;12:58-9.

12. Lynn SJ, Sawyers SM, Moller PW, O'Donnell JL, Chapman PT. Adultonset inflammatory myopathy: North Canterbury experience 1989-2001. Intern Med J 2005;35:170-3.

13. Baron M, Small P. Polymyositis/dermatomyositis: Clinical features and outcome in 22 patients. J Rheumatol 1985;12:283-6.

14. Koh ET, Seow A, Ong B, Ratnagopal P, Tjia H, Chng HH. Adult onset polymyositis/dermatomyositis: Clinical and laboratory features and treatment response in 75 patients. Ann Rheum Dis 1993;52:857-61.

15. Mastaglia FL, Phillips BA. Idiopathic inflammatory myopathies: Epidemiology, classification and diagnostic criteria. Rheum Dis Clin N Am 2002;28:723-42.

16. Troyanov Y, Targoff IN, Tremblay JL, Goulet JR, Raymond Y, Senécal JL. Novel classification of idiopathic inflammatory myopathies based on overlap syndrome features and autoantibodies: Analysis of 100 French Canadian patients. Medicine 2005;84:231-49.

17. Hengstman GJ, Brouwer R, Egberts WT, Seelig HP, Jongen PJ, van Venrooij WJ, \& al. Clinical and serological characteristics of 125 Dutch myositis patients: Myositis specific autoantibodies aid in the 
differential diagnosis of the idiopathic inflammatory myopathies. J Neurol 2002;249:69-75.

18. Ramanan AV, Feldman BM. Clinical features and outcomes of Juvenile Dermatomyositis and other childhood onset myositis syndromes. Rheum Dis Clin N Am 2002;28:833-58.

19. van der Meulen MF, Bronner IM, Hoogendijk JE, Burger H, van Venrooij WJ, Voskuyl AE, đal. Polymyositis: An over diagnosed entity. Neurology 2003;61:316-21.

20. Dalakas MC. Progress in inflammatory myopathies: Good but not good enough. J Neurol Neurosurg Psychiatry 2001;70:569-73.

21. Dalakas MC. Polymyositis, dermatomyositis and inclusion-body myositis. N Engl J Med 1991;325:1487-98.

22. Pestronk A, Kos K, Lopate G, Al-Lozi MT. Brachio-cervical inflammatory myopathies: Clinical, immune and myopathologic features. Arthritis Rheum 2006;54:1687-96.

23. Hill CL, Zhang Y, Sigurgeirsson B, Pukkala E, Mellemkjaer L, Airio A, $\notin$ al. Frequency of specific cancer types in dermatomyositis and polymyositis: A population-based study. Lancet 2001;357:96-100.

24. Dalakas MC. Muscle biopsy findings in inflammatory myopathies. Rheum Dis Clin N Am 2002;28:779-98.

25. Hoffman EP, Rao D, Pachman LM. Clarifying the boundaries between inflammatory and dystrophic myopathies: Insights from molecular diagnostics and microarrays. Rheum Dis Clin N Am 2002;28: $743-57$.

26. Gallardo E, Rojas-García R, De Luna N, Pou A, Brown RH Jr, Illa I. Inflammation in dysferlin myopathy: Immunohistochemical characterization of 13 patients. Neurology 2001;57:2136-8.

27. Greenberg SA, Sanoudou D, Haslett JN, Kohane IS, Kunkel LM, Beggs $\mathrm{AH}$, et al. Molecular profiles of the inflammatory myopathies. Neurology $2002 ; 59: 1170-82$

Accepted on 21-09-2008

Source of Support: Nil, Conflict of Interest: None declared. 